\title{
A Study on Enhanced Cross-Layering Handover Scheme for Supporting Seamless Routing to PMIPv6 Networks
}

\author{
Seungyong Shin ${ }^{1}$, Seungji Yu ${ }^{1}$, Sehee Maeng ${ }^{1}$, Changseop Song ${ }^{1}$ and Byungjoo Park ${ }^{1+}$ \\ ${ }^{1}$ Department of Multimedia Engineering, Hannam University, Ojeong-Dong, Daeduk-Gu, Korea.
}

\begin{abstract}
Proxy Mobile IPv6 (PMIPv6) based on network mobility has been suggested to reduce handover time in current methods and mobility management load of mobile phone. PMIPv6 decreases load of mobile phone as doing mobility management conducting on mobile phone in Network Agent and can reduce a mobility management delay time that may occur on fast handover and mobile phone. In this paper, It proposes an interworking of WIMAX L2 layer and PMIPv6 L3 layer, and a cross-layering fast handover method that suggests a support message to minimize a handover delay time when applying PMIPv6 networkbased IPv6 mobile protocol that standardizes in IETF to the WIMAX network.
\end{abstract}

Keywords: PMIPv6, WIMAX, Cross-Layering, Binding Update, Route Optimization

\section{Introduction}

In recent years, an information acquirement and use utilizing Internet by supply activation of high-speed Internet and portable devices are increasing rapidly. Mobile phone has been gradually made smaller and high-performance and Internet user's demands on the move that want to receive all kinds of high speed Internet services regardless of time and place are growing as wireless mobile telecommunication technologies develop. Active research is being done to provide these seamless services through Internet.

When wireless mobile node moves to new network, it is supportable a mobility by binding node's Home Address(HoA) in original network and Care-of Address(CoA) in transferred network to Home Agent(HA) through Mobile IPv6 protocol suggested in IETF (Internet Engineering Task Force) Mobile IP Working Group to support IP mobility. However, when node implements a handover, it needs processes Movement Detection, Address Configuration and Confirmation, Registration and a problem that may not receive the data that sent during this handover occurs. Also, there is a problem that before $\mathrm{CN}$ receives a binding update signal from MN with routing not optimized, it should continually take data packet through Home Agent(HA).

In the IETF, mobility management is recently not handled on node, and Proxy Mobile IPv6 (PMIPv6) treatable on the network is being standardized. PMIPv6 is that node can move to other wireless network without involving in handover as conducting movement related call processing signals which node should perform during handover in network agent. For these reason, it will be able to solve the battery and capacity problems of mobile node. As PMIPv6 finally manages mobility on the network, the mobility can be guaranteed by node without change of existing IPv6 stack.

In this paper, mobile node with on board IPv6 on the WIMAX network has optimized handover processes by defining an intimate interworking between PMIPv6 (L3 Layer) and WIMAX (L2 Layer) and a support message to minimize a handover delay time when subnet moves other area.

\section{Related Works and Problem}

\footnotetext{
Corresponding author. Tel.: +82(42)6298489; fax: +82(42)6298489.

E-mail address: bjpark@hnu.kr
} 


\subsection{Proxy Mobile IPv6 (PMIPv6)}

PMIPv6 (Proxy Mobile IPv6) has been standardized for a wireless network-based IP mobility processing in NetLMM (Network-based Localized Mobility Management) WG of IETF. If it uses the PMIPv6, node does not demand additional changes or renewal to manage mobility besides IPv6. As Operating System (OS) mounted on most current node basically supports IPv6 stack, those nodes which mount IPv6 stack receive automatically mobility service providing on the network without participating in IP mobility management. Mobile node which moves in PMIPv6 domain is identified as a MN-Identifier (e.g. NAI introduced in RFC 4283), and it can obtain Policy Profile from Policy Store.

If mobile node moves to New MAG(NMAG), it transfers its ID data in L2 handover procedure to the NMAG and requires authentication process for mobile node by sending to AAA server. By finishing an authentication process, MAG will acquire MN's profile, such as home address, home network prefix, Local Mobility Anchor (LMA) address and address configuration policy, necessary on the network when it happens handover. After that, MAG periodically transmits Router Advertisement (RA) Message to mobile node. Mobile node enters a RA message with each node's home network prefix for forming current connected link into home network. MAG forwards the Proxy Binding Update (PBU) message to LMA (home agent) to register new mobile node's location information. PBU message includes mobile node's ID and a home network prefix assigned to mobile node. LMA checks whether or not it has information about mobile node's ID in its binding cache entry through this message and if not having any information, it creates new information about mobile node. And then, it configures a tunnel between LMA and NMAG and sends Proxy Biding Acknowledgement (PBAck) message to MAG.

\subsection{WIMAX Handover Procedure}

Handover in WIMAX can be conceptually divided into adjacent network exploration, preparation process. Figure 1 shows the WIMAX handover procedure.

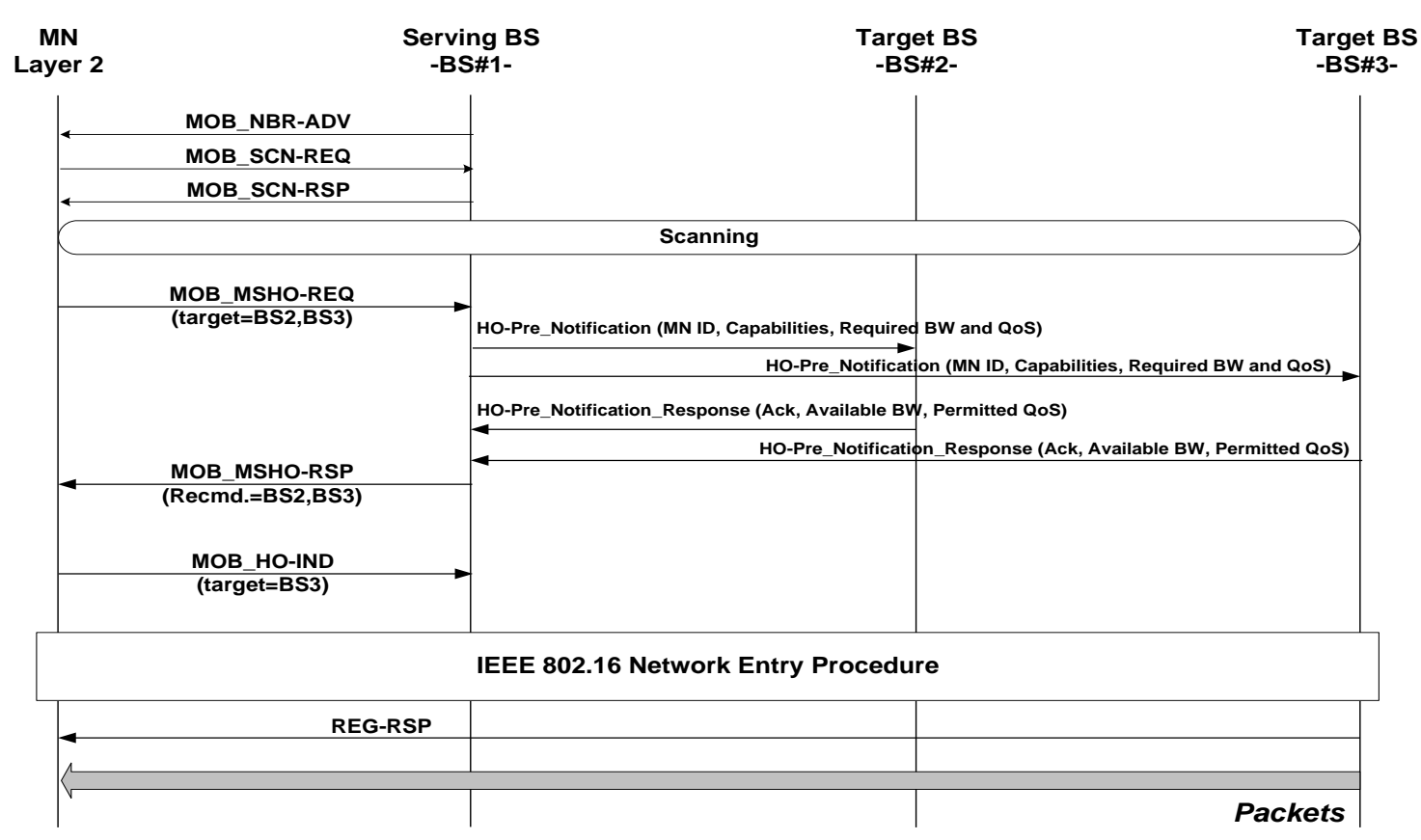

Fig. 1: WIMAX handover procedure.

WIMAX mobile node receives MOB_NBR-ADV (Neighbor Advertisement) message from Serving Base Station (Serving BS) which provides wireless connectivity equivalent to the network when it is located on any network. This message includes all BS data and relevant BS's network properties located in neighbor site. Therefore, if mobile node receives MOB_NBR-ADV message, it can use those data in future to more rapidly handover conduct by obtaining the ID, QoS (Quality of Service) parameter and channel information of adjacent BS. 
Another way to take network information is the process to measure downlink signal quality which receives from neighboring BS as a scanning procedure performed by mobile node. Mobile node gains adjacent BS's ID lists through MOB_NBR-ADV message and selects an appropriate BS based on real time link data received by scanning, and it can manage the lists as a candidate for BS to fulfil handover. Also, it is able to perform an association process with neighboring BS in scanning procedure to reduce the handover delay time.

Mobile node decides the most suitable Target BS to conduct handover according to standards, such signal strength and QoS parameter, which is received from neighbor BS which gets from former phase during handover preparation stage. Furthermore, in consideration of service qualities and signal intensity offered from current BS, mobile node determines handover and gives notice about this to the Serving BS(base station serving to a present mobile node on the relevant network of node). During this time, message is used as a Mobile Station Handover Request (MOB_MSHO-REQ) and transmitted to a Serving BS. And Serving BS responds including recommended BS's lists (candidate BS) to the Base Station Handover Response (MOB_BSHO-RSP) message. At this point Serving BS exchanges HO-pre-notification/HO-prenotification-response message with the candidate BS through backbone network, it can reduce the handover performance time by sending node's session and configural information for handover in advance.

If mobile node determines Target BS and is ready to move, it delivers this fact by sending Handover Indication (MOB_HOIND) message to the current Serving BS and implements handover. Mobile node cannot transmit and receive packet anymore through Serving BS since transmitting MOB_HO-IND message. Mobile node after move conducts network entry procedure. First of all, it synchronizes the link with Target BS as performing range and adjusts capability with Target BS if range is successfully completed. Then, Mobile node finally registers to Target BS through authentication. At this time, if Target BS receives node's data about authentication and capability through backbone network, mobile node can shorten handover procedure by skipping corresponding process. Lastly, if registration and entry process are finished successfully, Target BS is possible to start IP service to mobile node since then.

\section{Cross-Layering Handover Scheme for Supporting Seamless Routing to PMIPv6 Networks}

In this chapter, it describes the WIMAX network structure taken into account when network-based fast IPv6 handover method is applicable and the suggested method by referring to the message.

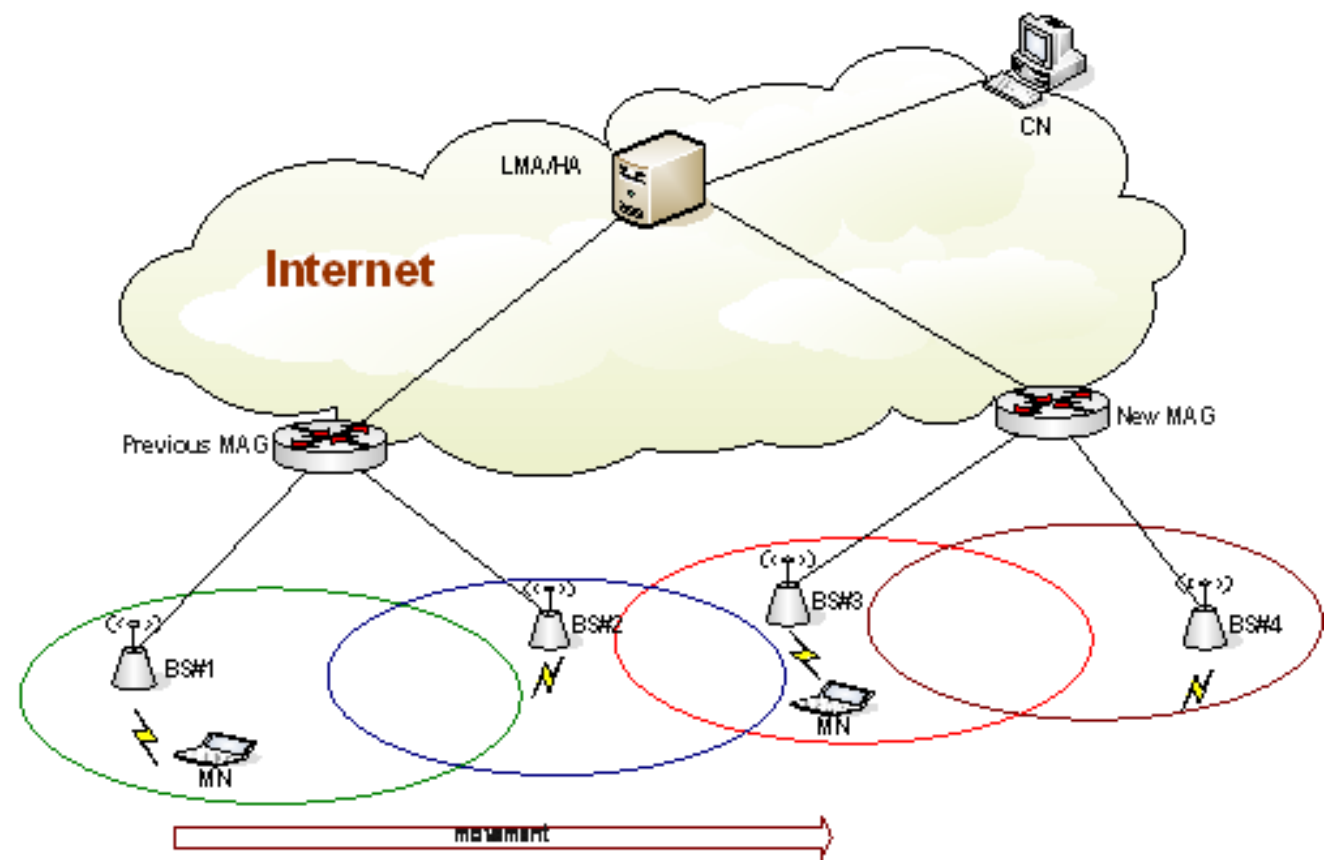

Fig. 2: Structure of WIMAX Networks

Figure 2 shows the structure of WIMAX networks. Network is divided into two subnets represented as a MAG in the figure, and one MAG manages a number of BS. In this case, the move between all BS does not 
need to handle IPv6 mobility all the time. When there is a move between BS in any MAG, it can keep communication by using only the handover according to WIMAX standard without IPv6 mobility management due to the move in same subnet. But as the case moving from BS1 to BS3 in figure 2, IPv6 mobility management and handover should be supported to maintain session in the situation transferring to new subnet.

\subsection{A proposed Handover Procedure}

In A fast handover operating sequence suggested in this thesis is shown by figure 3 .

(1) Like the WIMAX network, mobile node can get the surrounding BS's information, which are ID, QoS (Quality of Service), parameter and channel, through MOB_NBR-ADV to broadcast at regular intervals in Serving BS.

(2) Mobile node can conduct the Scanning about adjacent BS to take more detailed link information. It sets lists about Target BS to do handover based on the gained data, and sends a request for handover to Serving BS through MOB_MSHO-REQ.

(3) As Serving BS using a backbone network with Target BS communicates, it informs node's ID and a required Capability, Bandwidth, QoS, and sends MOB_MSHO-RSP to mobile node. Also it forwards HONOTIF message to PMAG including the data of Target BS and node's MAC address. Since then, PMAG which received message starts to buffer packet which is being transmitted from $\mathrm{CN}$.

(4) PMAG establishes the tunnel for packet transmission by exchanging HO-Initiation (Handover Initiation) and HO-Ack (Handover Acknowledgement) messages with NMAG before sending a HO-NOTIFAck message.

(5) Packet to mobile node becomes tunnelling to new NMAG from the moment PMAG receives HO-Ack.

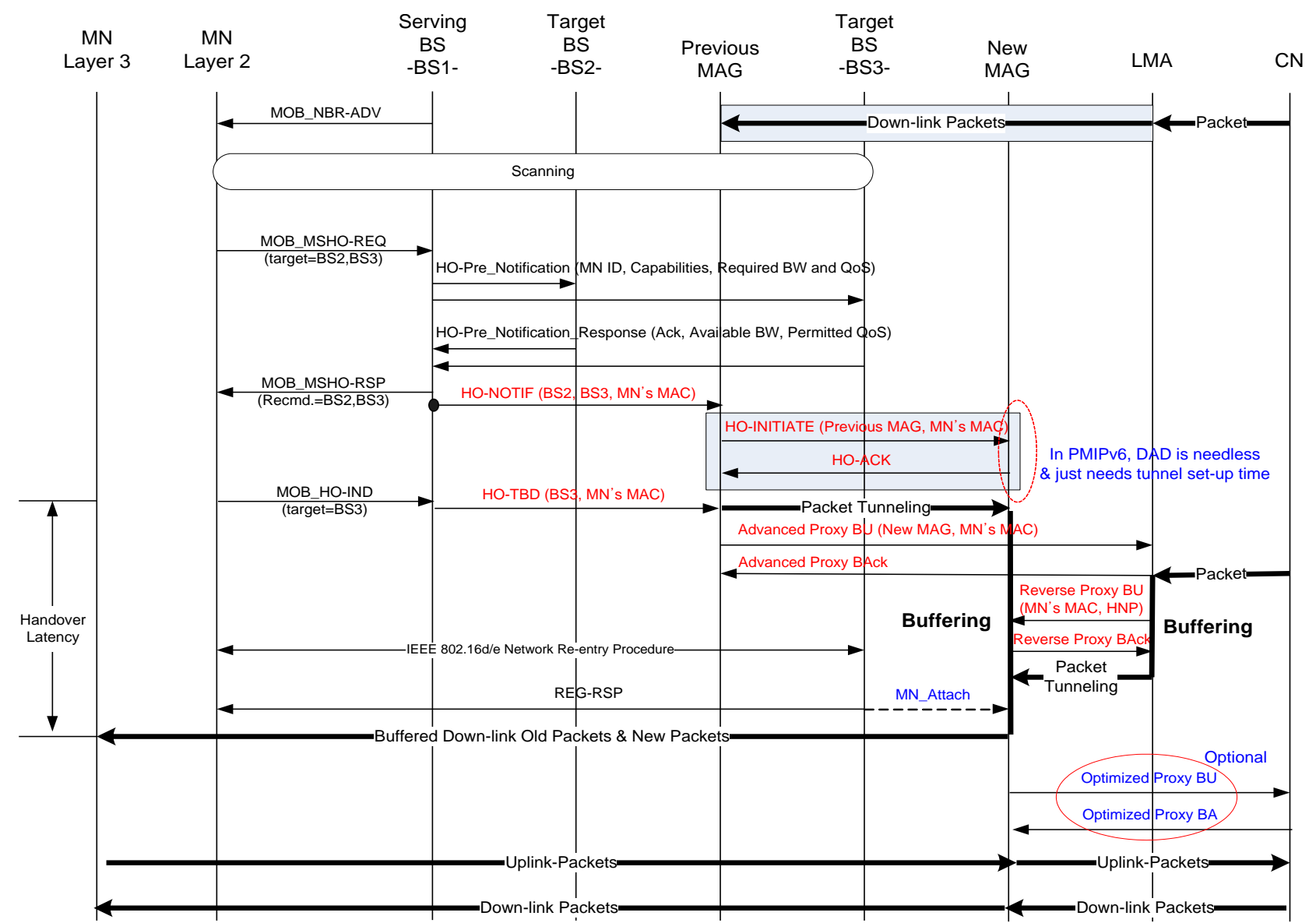

Fig. 3: Network-based Fast IPv6 Cross-Handover Message Exchange Procedure in WIMAX Networks 
(6) Serving BS which receives MOB_HO-IND sends HO-TBD message to PMAG involving Target BS's data and MAC address included in message. Since then, PMAG starts tunnelling for saved packet, and begins buffering about received packet at NMAG.

(7) PMAG exchanges Advanced Proxy BU/Advanced Proxy Binding Acknowledge messages to LMA to minimize tunnelling for packet transmitted from $\mathrm{CN}$.

(8) As soon as LMA sends Advanced Proxy Back message, it transmits information to LMAG including the MAC address and HNP(Home Network Prefix) of mobile node in Reverse Proxy BU message.

(9) After LMA receives Reverse Proxy Back message from NMAG, the buffering packet begins tunnelling to NMAG without having to pass through PMAG.

(10) WIMAX Network Re-entry procedure is finished as Target BS sends the REG-RSP, WIMAX message, to node. At this time, Target BS informs that mobile node makes newly connections with itself to NMAG connected to Target BS.

(11) At previous step, NMAG which receives notification for connection with mobile node transmits to packet buffered from PMAG and NMAG in the meantime. If NMAG supports PMIPv6 stack for tunnelling with NMAG to $\mathrm{CN}$, it can support a triangle routing problem which is passed by LMA through the exchange of Optimized Proxy BU/Back message defined as a option.

If the procedure stated above is used, mobile node can finish the WIMAX handover and receive data packet from NMAG at the same time. Therefore, L3 handover delay time of all handover delay time is able to be minimized, even though mobile node would be based on PMIPv6 mobility protocol.

\section{Conclusion}

A method has been suggested to aid fast handover on WIMAX network based on Proxy Mobile IPv6, network-based handover support protocol in this thesis. In the suggested method, mobile node can minimize responsibility for the battery efficiency, CPU processing time and stack change by not participating in a signalling related to handover and mobility. By Using IAPP and ND procedures for a seamless fast handover support based on PMIPv6 in WIMAX network, a measure to optimize the packet transmission is now investigating when mobile node moves to a neighbouring MAG in special environment composed of multiple MAG, and part of suggested methods through IETF contribution is being introduced.

\section{Acknowledgements}

This research was supported by Basic Science Research Program through the National Research Foundation of Korea (NRF) funded by the Ministry of Science, ICT and future Planning (2015R1A2A2A03002851).

\section{References}

[1] S. Gundavelli, K. Leung, V. Devarapalli, K. Chowdhury, B. Patil, "Proxy Mobile IPv6," IETF Netlmm, Internet Draft, March, 2007.

[2] Youn-Hee Han, Byungjoo Park, "A Fast Handover Scheme in Proxy Mobile IPv6," draft-han-netlmm-fastpmipv6-00 (work in progress), July, 2008.

[3] IEEE Std 802.16-2004, “IEEE Standard for Local and Metwropolitan area networks," June, 2004.

[4] Jinsoo Park, Dong-Hee Kwon and Young-Joo Suh, "An Integrated Handover Scheme for Fast Mobile IPv6 over IEEE 802.16e Systems,” Proceeding of IEEE Vehicular Technology Conference, Sep., 2006. 